\title{
Load carriage analysis for military using functional data analysis technique : registration and permutation test
}

\author{
W. R. Wan Din ${ }^{1 *}$; A. S. Rambely ${ }^{2}$; A. A. Jemain ${ }^{2}$ \\ ${ }^{l}$ Centre of Foundation Studies and Applied Sciences, National Defence University of Malaysia, 57000 Kem Sg Besi, \\ Kuala Lumpur, Malaysia \\ ${ }^{2}$ School of Mathematical Sciences, Faculty of Science \& Technology, The National Universiti of Malaysia, 46300 Bangi, Malaysia \\ *Corresponding author E-mail: wwanrozita@upnm.edu.my
}

Copyright $\odot 2014$ W. R. Wan Din et al. This is an open access article distributed under the Creative Commons Attribution License, which permits unrestricted use, distribution, and reproduction in any medium, provided the original work is properly cited.

\begin{abstract}
This paper focused on the analysis of ground reaction force (GRF) of load carriage for military through functional data analysis (FDA) technique. The main objective of the study is to investigate the effect of $10 \%$ bodyweight (BW) load increment for normal walking through FDA method. The experiment on GRF was carried out by ten healthy military soldiers aged $31 \pm 6.2$ years old; weighing $71.6 \pm 10.4 \mathrm{~kg}$ with the height of $166.3 \pm 5.9 \mathrm{~cm}$. Data was captured using Vicon 1.4 motion analysis system, Kistler force plates and thirty nine body markers attached to the subjects. FDA method mainly B-spline basis formation, time normalization, smoothing, curves registration and permutation tests were applied to analyze the data. The results confirmed that FDA did provide a better technique of smoothing to produce quality data in an easy manner. From this paper we conclude that registration did significantly align all the curves according to curves features providing a better way of distinguishing the amplitude of load increment. Finally, permutation tests confirmed that $10 \% \mathrm{BW}$ load increased from $0 \% \mathrm{BW}$ to $40 \% \mathrm{BW}$, have significant effect on soldiers regarding their load carriage and GRF.
\end{abstract}

Keywords: Military Load Carriage; GRF; FDA; Curve Registration; Permutation Test

\section{Introduction}

The paper deals with load carriage analysis using functional data analysis technique (FDA). The main objectives of this paper are to analyse the GRF parameter using FDA technique as it is a relatively new effective method available and also the study of the effect of $10 \%$ BW load increment on Malaysian soldiers. As far as the author is concerned, researches regarding load analysis have never been analysed using this method. In addition, no such study has been carried out regarding Malaysian military subject to the matter discussed. Researchers conducted regarding load analysis include load magnitude, spine injury and posture [8], [20], [23], effects of load distribution [5], load posture and gait [2], load and GRF [3],[13], rifle carriage and human gait [4], load carriage effects on lower limb [29], soldiers load carriage [15], determining optimum load [10], [22], postural sway [11], load and walking gait [12], [17], [18] and load and trunk movements [16], [21]. Malaysian military soldiers with usually smaller build-up size without exception have to carry heavy load up to $50 \mathrm{~kg}$ or more. Height for Malaysian soldier is normally around $165 \mathrm{~cm}$ and weight around 70 kg. Excessive load can cause spine injury [23] or perhaps more serious problems when they are getting older. This paper tries to investigate whether a $10 \% \mathrm{BW}$ affects Malaysian military as they have to carry excessive loads while training and also on battle ground. FDA has been applied to various fields such as in biology and chemistry [28], plankton analysis [14], lactate curves in Biology and Medicine [19], gait analysis [27] and kinematic data [6]. Other methods like one-way ANOVA [22], one-way MANOVA [3], principal component analysis [17] and many more have been used in analysing load data but not FDA. Prior to this paper, an analysis for trial experiment on load GRF data by using FDA has been completed [7]. FDA special features rely on its ability to transform data to functional forms for further steps of the analysis. Higher derivatives products can be produced through this method. Some other unique 
features in FDA that have been applied in the study are tools for smoothing data which are discussed thoroughly in the first part of the paper, landmark or curve registration that enable curves to be aligned according to phase or amplitude variation. Finally, permutation test is used to confirm the significance of $10 \%$ BW load increment to Malaysian soldiers. Besides these features, many more applications are available in order to analyse data such as functional principal and canonical components analysis, linear models for scalar and functional responses and also functional models and dynamics.

\section{Methodology}

\subsection{Data acquisition}

Ten healthy males, who work as military soldiers, aged $31 \pm 6.2$ years old, weight $71.6 \pm 10.4 \mathrm{~kg}$ with height of $166.3 \pm$ $5.9 \mathrm{~cm}$ had volunteered to participate in the experiment. Since all of them are soldiers, they have experienced carrying heavy load while training or in service. Hence, they did not encounter any problems while performing the experiment. Instructions and few trials were given beforehand to familiarize participants with the procedure needed. Two to three minutes rest time were given in between different load changes to avoid fatigue. The exercises were not much different from the trial experiment [7] except for the number of subjects and outfit. Previously, the subject wore full military uniform together with boots. The trial result proved that a lot of missing data and inaccurate position of markers were due to the wearing of uniform. Therefore, in this experiment the subject wore tight outfit without boots and carried normal military backpack with different loads. Thirty-nine body markers were attached to body joints as depicted in Fig. 1. He was then required to walk at normal walking pace along a six meter path where force platforms were located for every load experimented. The right feet touched the first force platform and the left touched the second platform.

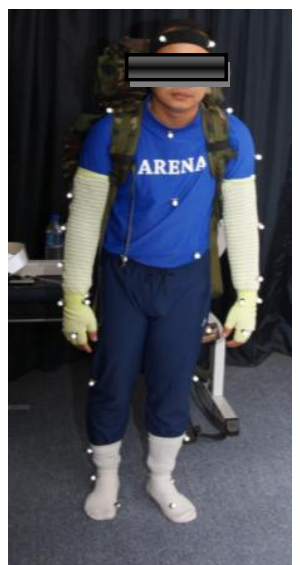

Fig. 1: A Subject Holding a Military Backpack with 37 Body Markers Attached To Joints

Loads consisting of normal military stuff such as food, blanket, cloth, additional books, etc. weighing $0 \%$ (control), $10 \%, 20 \%, 30 \%$ and $40 \%$ of each individual participant body weight (BW) were carried close to body centre of mass (CoM). The backpack weight was included together in the percentage of load to be carried. The subject walked five times in the same direction with each load while gait kinematics and kinetic data were being recorded using Vicon 1.4 Motion Analysis Systems comprised of seven cameras and two units of Kistler force plates. Ground reaction force (GRF) data were recorded by forces exerted by the two force platforms. Both platforms gave almost identical results after graphing the raw data, hence only data from the first platform that have foot struck fully on the force platforms were chosen for further analysis. Amount of data for every load consisted of forty four to forty nine trials.

\subsection{Data analysis}

Data recorded were resampled at $50 \mathrm{~Hz}$. Since we are interested to know the biomechanics aspects of the military gait and load carriage, parameters collected were GRF and trajectories parameters of coordinates in $\mathrm{x}, \mathrm{y}$ and $\mathrm{z}$ directions, angles for everybody segments, forces, moments and powers. GRF data was in the form of normal "M" shape when plotted (Fig. 2). Hence, the most suitable method to analyse this kind of data would be transforming it to functional form instead of using normal statistical distribution practice. FDA could provide us the tools of converting this data to functions followed by normal statistical verification. All trial cycles end at different period which are normal and expected as the load and subject varies in weight, height and body feature. Time normalization was performed to standardize the walking cycle to common values. Comparison of curves features later will be based on the same common percentage of the cycle. Even though Woltring filter has been applied, spikes still occur whereby smoothing is mandatory. 
Sets of basis functions together with their coefficients were used to construct functional data objects. Since the data were not periodic functions, (again as performed in trial data analysis) [7]. B-spline basis expansion was selected to represent the curve in the form of functional data object. The model takes the form,

$$
x(t)=\sum_{k=1}^{K} c_{k} \phi_{k}(t)
$$

Where $c_{k}$ are the corresponding coefficients and $\phi_{k}(t)$ is a set of B-spline basis functions [25]. In this case, it was done together with smoothing process using directly specified roughness penalty. The B-spline functions used were polynomials basis functions with order six. The polynomials segments of basis function were jointed at specified knots or break points strictly increasing and smoothed which was the length of the individual curves. Degrees of freedom for these particular curves were the number of interior knots excluding lower and upper limits plus the order of polynomials. Smoothing parameters applied were by roughness penalty, penalizing fourth derivatives, with lambda 1e12 amount of smoothing to be applied to estimated functional parameters. Further explanation on constructing basis function is available in [26].

The following step was landmark or curve registration whereby all the curves were aligned according to their peak and trough varying in amplitude. The process started with finding the precise points for peaks and valleys for all the curves where the registration was to be done. The function used timings of these features to estimate a non-linear transformation of the argument continuum for each functional observation called warping function [24]. For warping and registration purposes, the B-spline functions constructed were polynomials of ten basis functions with order six. The polynomials segments of basis function were jointed at seven specified knots or break points strictly increasing and smoothed. Degrees of freedom for these particular curves were the number of interior knots excluding lower and upper limits plus the order of polynomials which were thirteen altogether. Time-warping functions must be strictly increasing, differentiable at points of interest and must satisfy the constraints $h(0)=0$ and $h(T)=T$ where $(0, T)$ is the curve interval. The registered height function or amplitude in this case is

$$
x_{i}^{*}(t)=x_{i}\left[h_{i}^{-1}(t)\right],
$$

and the aligning function $h^{-1}(t)$ must satisfy the functional inverse of $h(t)$,

$$
h^{-1}[h(t)]=t
$$

Registration was the most important process in the analysis sequence since it could align all the prominent features of the curves which previously is not possible through other methods beside FDA. Only by applying this process, curves of different loads could be distinguished easily which is not possible before.

Permutation test was selected to test the significance of the testing load increment. It is usually as powerful as or more powerful than alternative approaches [9] like bootstrapping for instance. Permutation tests were carried out on two different purposes. Firstly, test the significance of increasing the load every $10 \% \mathrm{BW}$ and secondly test the effect of curve registration in the overall process flow. Therefore it was done twice, after smoothing and again after curve registration with results displayed side by side for comparison as in Fig. 3. The test done was the t-statistics calculated point wise and the test was based on the maximal value with default of 101 equally-spaced points. It first shuffles the labels of the curves before calculating the maximum of $t(x)$ with the new labels [11]. It is hypothesized that there is no difference between two mean samples. The sample here refers to load tested. The null hypothesis testing is therefore no difference between the two population means and alternative hypothesis testing is the population means are different,

$$
\begin{aligned}
& \mathrm{H}_{\mathrm{o}}: \mu_{1}=\mu_{2} \\
& \mathrm{H}_{\mathrm{a}}: \mu_{1} \neq \mu_{2}
\end{aligned}
$$

T-statistics equation takes the form of

$$
t=\frac{\bar{X}_{1}-\bar{X}_{2}}{\sqrt{\frac{S_{1}^{2}}{n_{1}}+\frac{S_{2}^{2}}{n_{2}}}}
$$

Where $\bar{X}$ the sample is mean, $S$ is the standard deviation and $\mathrm{n}$ is the sample size for every load calculated individually. Two adjacent groups of loads were compared i.e. $0 \%$ BW vs. $10 \%$ BW, 0\% BW vs. 20\% BW, 20\% BW vs. $30 \% \mathrm{BW}$ and $30 \% \mathrm{BW}$ vs. $40 \% \mathrm{BW}$.

The programming of statistical analyses was done in $\mathrm{R}$, which is also available in S-Plus and Matlab. FDA package which is free online was used extensively in the analyses. 


\section{Result and Discussion}

\subsection{Raw data, time normalization and smoothing}

GRF curves captured from all trials of different loads yield the normal gait curve shape "M". Fig. 2 depicts two out of five GRF curves which are $0 \% \mathrm{BW}$ load which act as control and $10 \% \mathrm{BW}$ load. Gait cycle for different loads and subjects varies in phase and amplitude. Obviously it is impossible to compare between the first and second loads by observing those graphs. There exist phase shifts with all peaks and valleys occur at slightly different times.
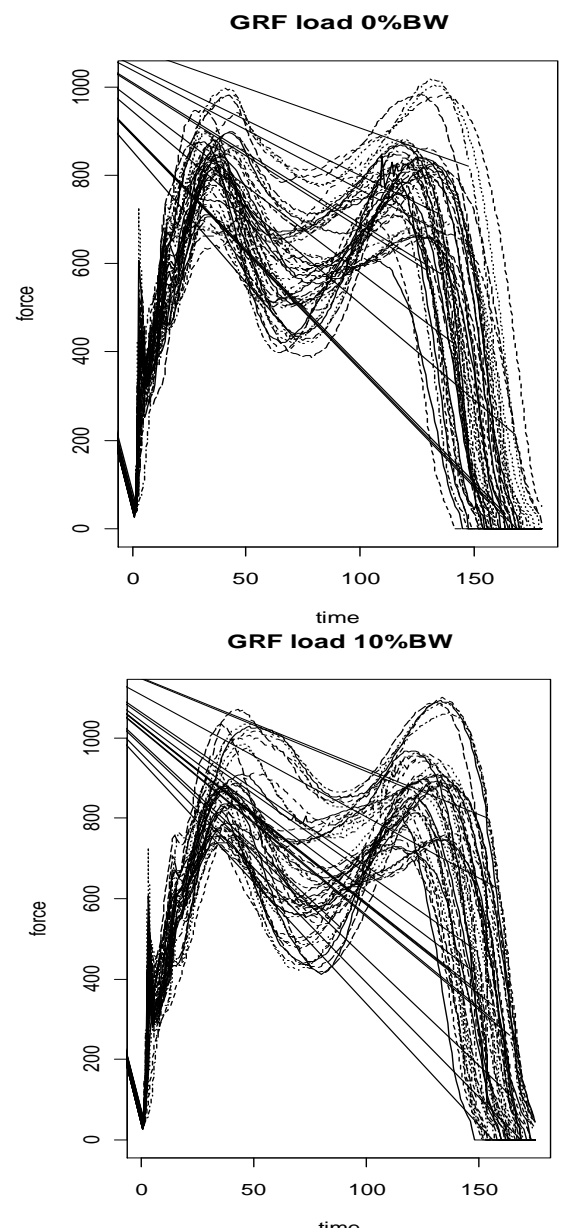

Fig. 2: GRF for All Trials before Time Normalization for Load 0\% BW and 10\% BW.

Walking gait cycle time period varies for different loads as in Table 1. Increasing the load had increased the period taken for each cycle, load of $0 \% \mathrm{BW}$ starts at $148 \mathrm{sec}$ and ends at $184 \mathrm{sec}$ whereby load 10\% BW starts slightly later at $151 \mathrm{sec}, 20 \% \mathrm{BW}$ at $153 \mathrm{sec}, 30 \% \mathrm{BW}$ at $155 \mathrm{sec}$ and $40 \% \mathrm{BW}$ at $163 \mathrm{sec}$. An increase of $10 \%$ BW delays the start cycle time by two to three seconds for every load.

\begin{tabular}{lll}
\multicolumn{3}{c}{ Table 1: Range of Gait Cycle for All Military Loads Change. } \\
\hline Load & Start gait cycle (sec) & End gait cycle (sec) \\
\hline $0 \%$ BW & 148 & 184 \\
$10 \%$ BW & 151 & 175 \\
$20 \%$ BW & 153 & 184 \\
$30 \%$ BW & 155 & 194 \\
$40 \%$ BW & 163 & 194 \\
\hline
\end{tabular}

Time normalization is able to standardize the gait cycle to a common value and reduce phase shifting at the end of the cycle. Somehow, it is still impossible to compare the amplitude variation occurred. Previously in the trial data experiment [7], since the amount of data collected is limited, time normalization alone is considered adequate without having to go through registration phase. Somehow, it proved irrelevant after actual data is collected where we observed that time normalization alone is not sufficient to compare the result. FDA registration and warping function approach 
can overcome this problem by aligning the curves according to features of interest. In this case, we align all the curves at three main points where maximum and minimum are located.

\subsection{Registration and warping}

Registration is used to separate the variation in timing of maximum and minimum GRF occurrences. The phase at the beginning of one GRF curve to another is out by few frames or seconds. Registration is applied to shift every curve such that every one of them starts at the same moment resulted in zero phase lag. Fig. 3 depicts the result of registration and warping functions for each load. The first column is the smoothed data without time normalization and registration, while the second column is the respective warping functions to be used for registration purposes. The third column is the result of the data after smoothing, time normalization and registration. By comparing the first and third columns, registration does tremendously improve the data whereby phase shifting has been eliminated resulting in only amplitude variation for comparison. The highest and lowest peaks and valleys are clearly visible. In order to verify the difference in amplitude variation, mean for every load is calculated. Fig. 4 clearly displays the mean GRF for the five loads experimented with information tabulated in Table 2. From the graph, there exists a certain amount of differences in the magnitude of GRF for every load increment. Load $0 \% \mathrm{BW}$ is the lowest curve at the bottom which conveys the GRF of a normal military while walking without carrying any load which is equal to GRF of a normal person, control BW of a military subject. The first peak where heel strikes the force plate recorded $813.55 \mathrm{~N}$ GRF at $21 \%$ of the walking gait cycle for load $0 \% \mathrm{BW}$. Minimum GRF of $583 \mathrm{~N}$ occurs at $40 \%$ of the gait cycle and second maximum is $774.94 \mathrm{~N}$ at $67 \%$ of the gait cycle. Increasing the load by $10 \% \mathrm{BW}$ has resulted in an increase of the first peak GRF by $4 \%$ to 847.13 $\mathrm{N}$, the minimum curve also increases by $9 \%$ to $635.63 \mathrm{~N}$ and the second peak increases by $9.6 \%$ to $849.67 \mathrm{~N}$. Mean GRF for $20 \% \mathrm{BW}$ is $904.3 \mathrm{~N}$ at the first peak which increased by $6.75 \%$ compared to that of the previous load, the minimum force is $694.16 \mathrm{~N}$ which is $9.21 \%$ more than $10 \% \mathrm{BW}$ and $929.68 \mathrm{~N}$ for the second peak which also increases by $9.42 \%$. Mean GRF for $30 \% \mathrm{BW}$ are $973.46 \mathrm{~N}$ for the first peak, $751.02 \mathrm{~N}$ for its minimum force and $994.31 \mathrm{~N}$ for the second peak with $7.65 \%, 8.19 \%$ and $6.95 \%$ increases for the first peak, minimum and the second peak respectively. The heaviest load which is $40 \% \mathrm{BW}$ recorded the highest mean GRF of $1011 \mathrm{~N}$ for the first maximum, $814.29 \mathrm{~N}$ for minimum force and $1069.4 \mathrm{~N}$ for the second maximum which are $3.92 \%, 8.42 \%$ and $7.55 \%$ increases for those three points respectively.

Table 2: Ground Reaction Force (GRF) Mean Values, Generalized Time and Percentage Load Increased for the First Peak (Heel Strike), Force Minimum and the Second Peak (Toe Off) for Load 0\% BW, 10\% BW, 20\% BW, 30\% BW and 40\% BW after Time Normalization and Registration.

\begin{tabular}{|c|c|c|c|c|}
\hline & & $\begin{array}{c}\text { First peak } \\
\text { (Heel strike) }\end{array}$ & Force Minimum & $\begin{array}{c}\text { Second peak } \\
\text { (Toe off) }\end{array}$ \\
\hline \multirow[t]{5}{*}{ Mean GRF } & $0 \% \mathrm{BW}$ & 813.55 & 583.78 & 774.94 \\
\hline & $10 \% \mathrm{BW}$ & 847.13 & 635.63 & 849.67 \\
\hline & $20 \% \mathrm{BW}$ & 904.30 & 694.16 & 929.68 \\
\hline & $30 \% \mathrm{BW}$ & 973.46 & 751.02 & 994.31 \\
\hline & $40 \% \mathrm{BW}$ & 1011.58 & 814.29 & 1069.36 \\
\hline \multirow{5}{*}{$\begin{array}{l}\text { Normalized } \\
\text { time }\end{array}$} & $0 \% \mathrm{BW}$ & 0.21 & 0.40 & 0.67 \\
\hline & $10 \% \mathrm{BW}$ & 0.22 & 0.44 & 0.70 \\
\hline & $20 \% \mathrm{BW}$ & 0.22 & 0.44 & 0.67 \\
\hline & $30 \% \mathrm{BW}$ & 0.21 & 0.42 & 0.65 \\
\hline & $40 \% \mathrm{BW}$ & 0.22 & 0.42 & 0.65 \\
\hline \multirow{4}{*}{$\begin{array}{c}\text { Percentage } \\
\text { Load } \\
\text { increased }\end{array}$} & $10 \% \mathrm{BW}$ & $4.13 \%$ & $8.88 \%$ & $9.64 \%$ \\
\hline & $20 \% \mathrm{BW}$ & $6.75 \%$ & $9.21 \%$ & $9.42 \%$ \\
\hline & $30 \% \mathrm{BW}$ & $7.65 \%$ & $8.19 \%$ & $6.95 \%$ \\
\hline & $40 \% \mathrm{BW}$ & $3.92 \%$ & $8.42 \%$ & $7.55 \%$ \\
\hline
\end{tabular}



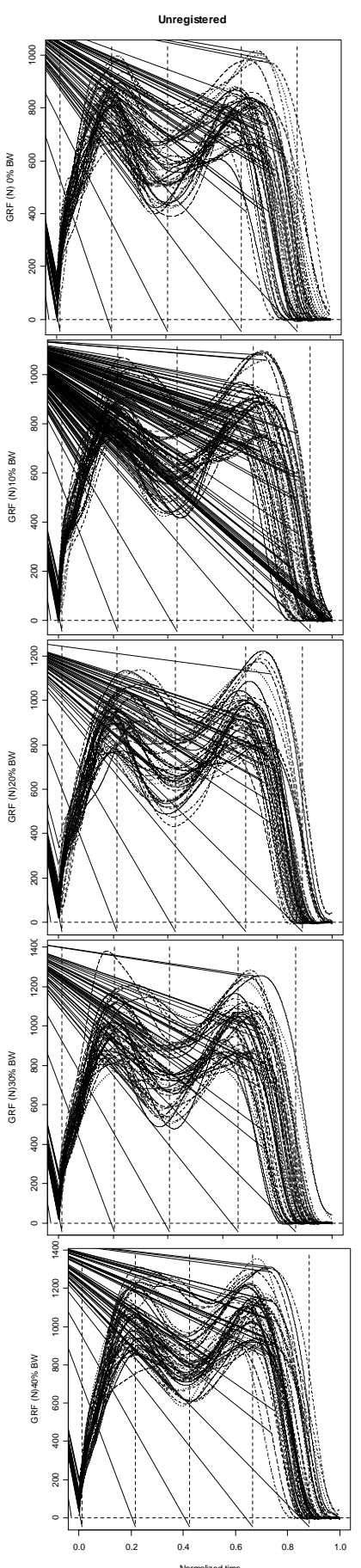
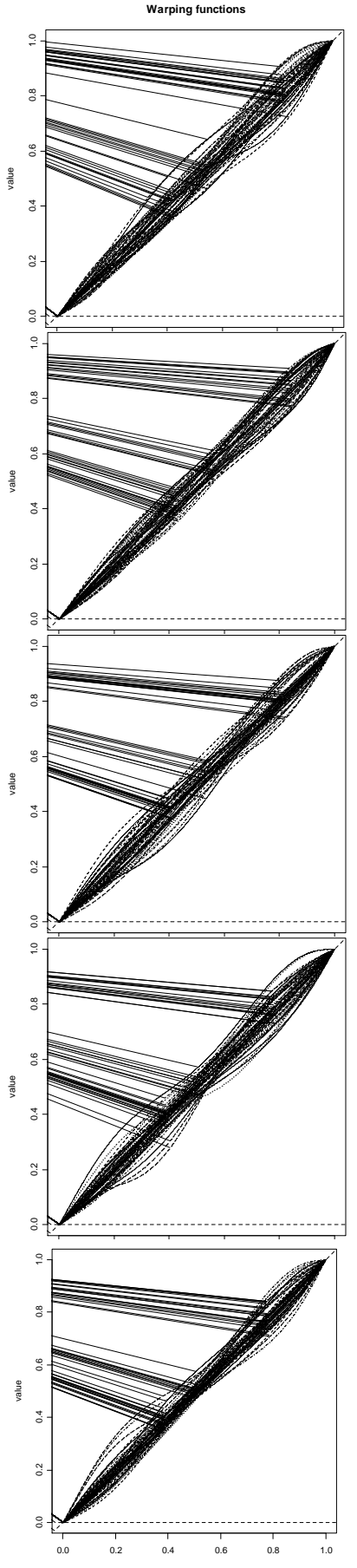
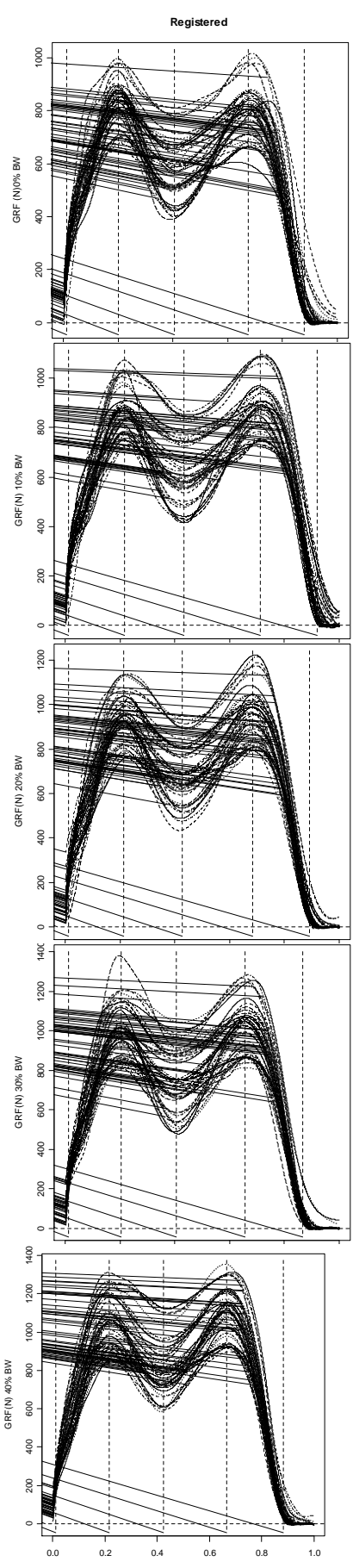

Fig. 3: Functional GRF Curves for Unregistered Data ( $\left(1^{\text {st }}\right.$ Column), Warping Functions ( $2^{\text {nd }}$ Column) and Registered Data ( $3^{\text {rd }}$ Column) for Load $0 \%$ BW, 10\% BW, 20\% BW, 30\% BW and 40\% BW Load Respectively

The next step is to verify whether this $10 \%$ BW load increment is significant to Malaysian military in particular. Permutation test is performed to identify the overall findings of the experiment towards Malaysian military load analysis. 


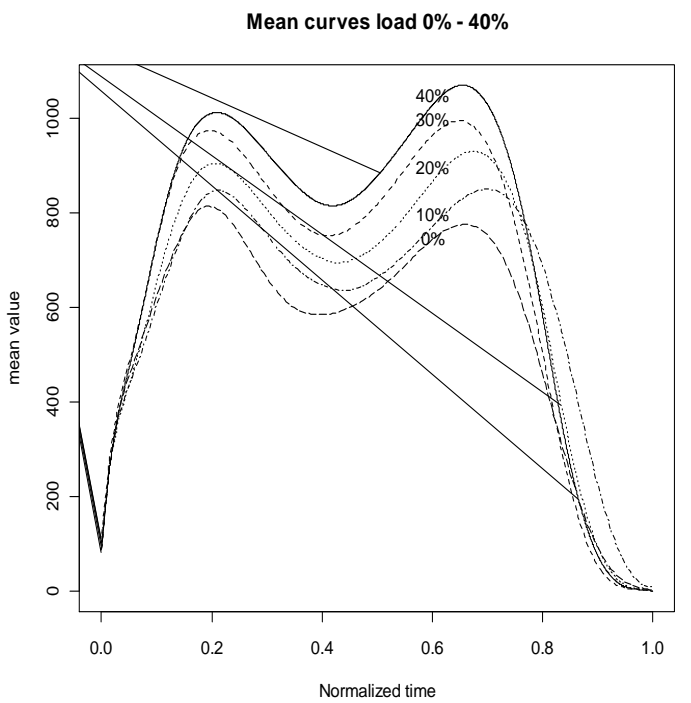

Fig. 4: Mean GRF Curves after Time Normalization and Registration for 0\% BW, 10\% BW, 20\% BW, 30\% BW and 40\% BW Loads Respectively.

\subsection{Permutation test}

Permutation test is done to determine whether $10 \% \mathrm{BW}$ load increment is statistically significant. Again as performed in the trial data experiment (Din. et al., 2011) sequence, permutation test is done before and after registration to test the significance of including registration in the process flow. The test to find out the significance of $10 \% \mathrm{BW}$ load increment is done by comparing $0 \% \mathrm{BW}$ to $10 \% \mathrm{BW}, 10 \% \mathrm{BW}$ is compared to $20 \% \mathrm{BW}, 20 \% \mathrm{BW}$ is compared to $30 \%$ BW and finally $30 \% \mathrm{BW}$ is compared to $40 \%$ BW. Fig. 5 displays the permutation tests results, the observed statistics, pairwise 0.05 critical values and maximum 0.05 critical values, which are illustrated side by side for comparison. Points where the observed statistics graph rise above the maximum 0.05 critical value are considered $95 \%$ statistically significant. From the displays, all the tests before and after registration recorded a $95 \%$ significant level which means the $10 \%$ BW load increment is significant to the Malaysian subjects. The first column of Fig. 5 depicts the results of permutation tests from unregistered data input. We can see that even before registration is applied, 10\% BW load increment can affect Malaysian soldiers as all the tests are significant. Minimum and maximum points in the data before testing (Fig. 2) are where the significant effects occur. However, the cycles of gait where it occurs are not the same compared to that after registration as we can see in displays of the second column of Fig. 5. The shape of the graphs from one test to another is not consistent without registration. Permutation tests after registration are more reliable and prominent as all the graphs are more or less similar in shape for all the comparison which means the body position where the data is significant is about the same gait cycle. Most of them are significant at those three minimum and maximum points of the original data.

\section{Conclusion}

GRF data is in the form of curves when plotted and it leads to the appropriate way of analyzing this kind of data which is not through finding its statistical distribution but by initially converting it to function. FDA is the most suitable method in handling this kind of data because of its ability to convert data into functional forms for further step of analyzing. Registration has proved to be the most significant process which enables the data to be aligned according to their main features. Phase shifting can be removed through this process which amplifies the amplitude variation according to load increment. Mean curves displays make the amplitude differences existence possible whereby we can distinguish the magnitude of GRF occurrence at the peak and trough accordingly. A $10 \%$ BW load increment recorded an increase of approximately $4 \%$ to $9 \%$ in GRF but not uniformly. In other word, percentage of mean increase is not proportional to $10 \% \mathrm{BW}$ load increment specifically for Malaysian soldiers in this context.

The permutation tests have proved that every $10 \% \mathrm{BW}$ load increment is significant at $95 \%$ level. Before and after registration apparently give different results. The results of the permutation tests before registration are significant for the first three of $0 \% \mathrm{BW}$ vs. $10 \% \mathrm{BW}, 10 \% \mathrm{BW}$ vs. $20 \% \mathrm{BW}$ and $20 \% \mathrm{BW}$ vs. $30 \% \mathrm{BW}$. The shape of graphs is also different after registration is applied. The last comparison of $30 \% \mathrm{BW}$ vs. $40 \% \mathrm{BW}$ is not significant without registration. This is contrary to the results of permutation tests after registration where all the tests are significant at the first peak, minimum force and the second peak with the same shape which mean the percentage of cycle time occurrence at 0.05 critical values are equivalent. Therefore, again it is justified that registration is recommended and 
should be included in the sequence and the permutation tests should be done after registration and not before registration is applied. The other conclusion by comparing previous pilot study [7] where limited number of trials were being analyzed using the same processing sequence is the time normalization whereby it is proved to be inadequate in handling phase shifting for data at least more than thirty trials. Again registration is exceptionally good at aligning phase. Permutation test might not be the best test for limited number of trial as in pilot study but it is confirmed the most appropriate for this experimented data.

Finally, we conclude that $10 \% \mathrm{BW}$ load increment is significant to Malaysian military soldiers and FDA is the best method in analyzing the data.
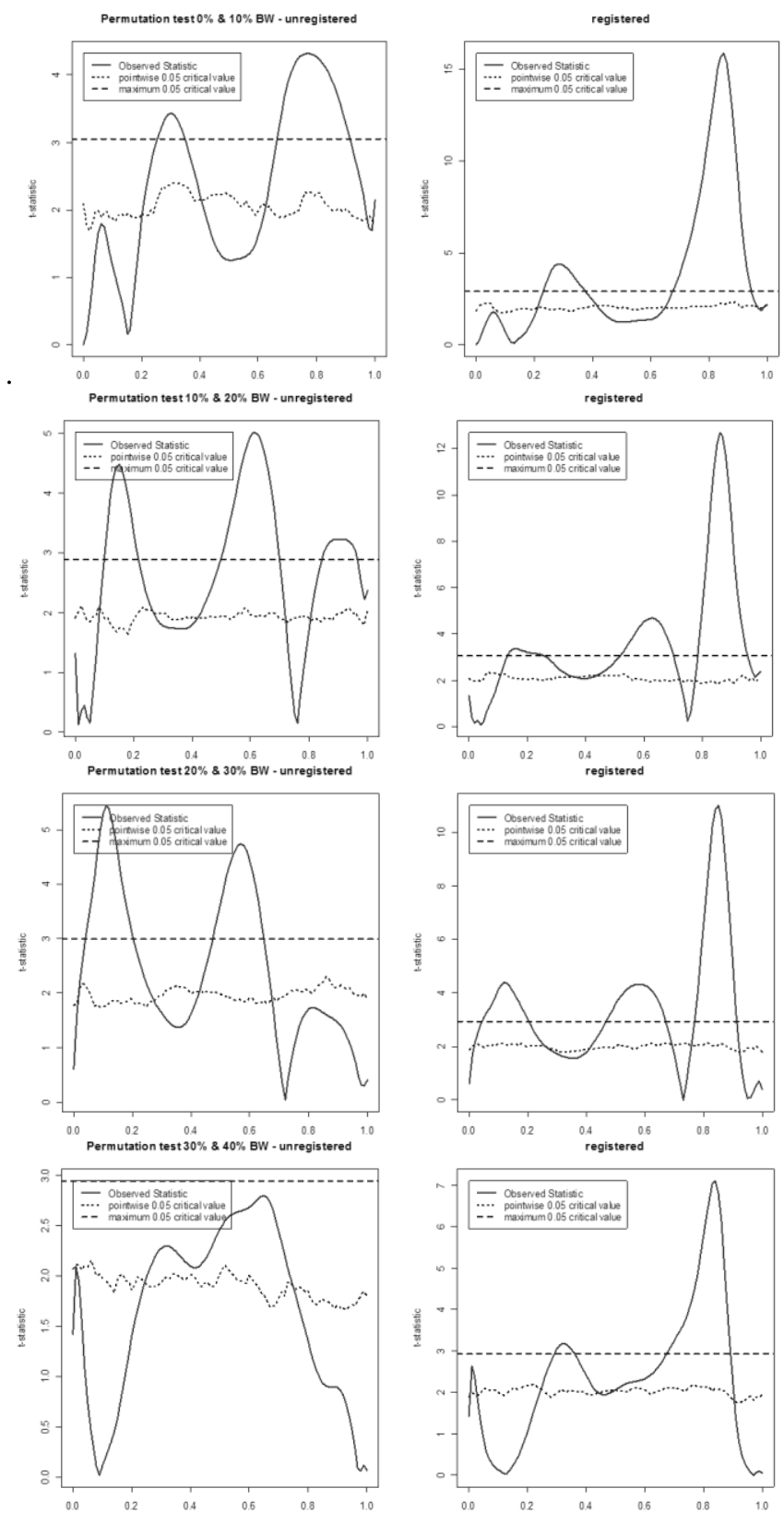

Fig. 5: Permutation Tests before (Left Column) and after (Right Column) Curves Registration Comparing $0 \%$ BW Vs. 10\% BW ( $1^{\text {st }}$ Row), 10\% BW Vs. $20 \%$ BW ( $2^{\text {nd }}$ Row $), 20 \%$ BW Vs. $30 \%$ BW ( $3^{\text {rd }}$ Row $)$ And $30 \%$ BW Vs. $40 \%$ BW ( $4^{\text {th }}$ Row $)$.

\section{Acknowledgements}

This work was supported by funding from UKM-OUP-NBT-28-135/2011and RAGS/UPNM/SG/04/4. We would like to thank University of Malaya, for providing the Motion Analysis Laboratory and meaningful discussion and suggestions. We also would like to thank PALAPES UKM for providing the military soldiers as the subject for this experiment and also valuable discussion regarding military procedure. 


\section{References}

[1] Y.S.S.M. Al-Khabbaz, T. Shimada, M. Hasegawa, The effect of backpack heaviness on trunk-lower extremity muscle activities and trunk posture, Gait \& Posture 28(2008) 297-302. http://dx.doi.org/10.1016/j.gaitpost.2008.01.002.

[2] R. L. Attwells, S. A. Birrell, R. H. Hooper, N. J. Mansfield, Influence of carrying heavy loads on soldiers posture, movements and gait, Ergonomics 49,14 (2006) 1527-1537. http://dx.doi.org/10.1080/00140130600757237.

[3] S. A. Birrell, R. H. Hooper, R. A. Haslam, The effect of military load carriage on ground reaction force, Gait \& Posture 26(2007) 611-614. http://dx.doi.org/10.1016/j.gaitpost.2006.12.008.

[4] S. A. Birrell, R.A. Haslam, The influence of rifle carriage on kinetics of human gait, Applied Ergonomics 51, 6(2008) 816-26. http://dx.doi.org/10.1080/00140130701811859.

[5] S. A. Birrell, R.A., Haslam, The effect of load distribution within military load carriage systems on the kinetics of human gait, Applied Ergonomics 41, 4(2010) 585-590. http://dx.doi.org/10.1016/j.apergo.2009.12.004.

[6] E. A. Crane, R. B. Cassidy, E. D. Rothman, G. E. Gerstner, Effect of registration on cyclical kinematic data, Journal of Biomechanics 43(2010) 2444-2447. http://dx.doi.org/10.1016/j.jbiomech.2010.04.024.

[7] W. R. Din, A. S. Rambely, A.A. Jemain, Load carriage analysis for Malaysian military using functional data analysis technique, In Proceedings of 4th International Conference on Modeling, Simulation \& Applied Optimization (ICMSAO), IEEE Xplore (2011) 1-8.

[8] J-H. Goh, A. Thambyah, K. Bose, Effects of varying backpack loads on peak forces in the lumbosacral spine during walking, Clinical Biomechanics 13, 1(1998) Supplement 1, S26-S31.

[9] P. Good, Permutation tests : A practical guide to resampling methods for testing hypotheses, Springer series in statistics, Springer-Verlag, New York, 1994 pp 1- 2. http://dx.doi.org/10.1007/978-1-4757-2346-5_1.

[10] M. F. Haisman, Determinant of load carrying ability, Applied Ergonomics 19, 2(1998) 111-121. http://dx.doi.org/10.1016/0003-6870 (88)90004-X.

[11] M. F. Heller, J. H. Challis, A. Neil, N. A. Sharkey, Changes in postural sway as a consequence of wearing a military backpack, Gait \& Posture 301 (2009) 115-117. http://dx.doi.org/10.1016/j.gaitpost.2009.02.015.

[12] K. Hiroshi, Effects of different loads and carrying systems on selected biomechanical parameters describing walking gait, Ergonomics 28 , 9(1985) 1347 - 1362. http://dx.doi.org/10.1080/00140138508963251.

[13] S. M. Hsiang, C. Chang, The effect of gait speed and load carrying on the reliability of ground reaction forces, Safety Science 40, 7-8(2002) 639-657.

[14] T. Ikeda, M. Dowd, J. L. Martin, Application of functional data analysis to investigate seasonal progression with interannual variability in plankton abundance in the Bay of Fundy, Canada, Estuarine, Coastal and Shelf Science 78(2008) 445-455. http://dx.doi.org/10.1016/j.ecss.2007.12.011.

[15] J. Knapik, E. Harman, K. Reynolds, Soldiers load carriage: Historical, Physiological, Biomedical, and Medical aspects, Applied Ergonomics 27, 3(1996) 207-216. http://dx.doi.org/10.1016/0003-6870 (96)00013-0.

[16] M. LaFiandra, R. C. Wagenaar, K. G. Holt, J. P. Obusek, How do load carriage and walking speed influence trunk coordination and stride parameters? Journal of Biomechanics 36, 1(2003) 87-95. http://dx.doi.org/10.1016/S0021-9290 (02)00243-9.

[17] M. H. Lee, M. Roan, B. Smith, An application of principal component analysis for lower body kinematics between loaded and unloaded walking, Journal of Biomechanics 42(2009) 2226-2230. http://dx.doi.org/10.1016/j.jbiomech.2009.06.052.

[18] Lei Ren, R. K. Jones, D. Howard, Dynamic analysis of load carriage biomechanics during level walking, Journal of Biomechanics 38, 4(2005) 853-863. http://dx.doi.org/10.1016/j.jbiomech.2004.04.030.

[19] J. Newella, K. McMillan, S. Grant, G. McCabe, Using functional data analysis to summarize and interpret lactate curves, Computers in Biology and Medicine, 36(2006) 262-275. http://dx.doi.org/10.1016/j.compbiomed.2004.11.006

[20] H. A. Orloff, The effects of load carriage on spinal curvature and posture, Spine 29, 12(2004) 1325-1329. http://dx.doi.org/10.1097/01.BRS.0000127185.74191.B1.

[21] R. S. Padula, H. J. C. G. Coury, Sagittal trunk movements during load carrying activities: a pilot study, International Journal of Industrial Ergonomics 32, 3(2003) 181-188. http://dx.doi.org/10.1016/S0169-8141 (03)00062-3.

[22] M. S. Pal, D. Majumdar, M. Bhattacharyya, R. Kumar, Optimum load for carriage by soldiers at two walking speeds on level ground, International Journal of Industrial Ergonomics 39(2009) 68-72. http://dx.doi.org/10.1016/j.ergon.2008.05.002.

[23] R. J. Parkinson, J. P. Callaghan, The role of dynamic flexion in spine injury is altered by increasing dynamic load magnitude, Clinical Biomechanics 24, 2(2009) 148-154. http://dx.doi.org/10.1016/j.clinbiomech.2008.11.007.

[24] J. O. Ramsay, G. Hooker, S. Graves, Functional Data Analysis with R and MATLAB, New York, Springer, 2009 pp 117-124. http://dx.doi.org/10.1007/978-0-387-98185-7_8.

[25] J. O. Ramsay, B. W. Silverman, Functional Data Analysis, Second Edition, Springer, New York, 2005 pp 127-145.

[26] J. O. Ramsay, B. W. Silverman, Functional Data Analysis, Second Edition. Springer, New York, 2005 pp 49-53.

[27] H. Sadeghi, P. Allard, K. Shafie, P. A. Mathieu, S. Sadeghi, F. Prince, J. Ramsay, Reduction of gait data variability using curve registration, Gait and Posture 12(2000) 257-264 http://dx.doi.org/10.1016/S0966-6362(00)00085-0.

[28] J. J. Song, W. Deng, H. J. Lee, D. Kwon, Optimal classification me-course gene expression data using functional data analysis, Computational Biology and Chemistry 32, 6(2008) 426-432. http://dx.doi.org/10.1016/j.compbiolchem.2008.07.007.

[29] D. C. Tilbury-Davis, H. H. Robin, The kinetic and kinematic effects of increasing load carriage upon the lower limb, Human Movement Science 18, 5(1999) 693-700. http://dx.doi.org/10.1016/S0167-9457(99)00026-3. 